\title{
A Digital Health Weight Loss Program in 250,000 Individuals
}

\author{
Conor Senecal $\left(\mathbb{D},{ }^{1}\right.$ Robert Jay Widmer $\mathbb{D}^{2},{ }^{2}$ Beth R. Larrabee, ${ }^{3}$ Mariza de Andrade $\mathbb{D}^{3}{ }^{3}$ \\ Lilach O. Lerman $\mathbb{D}^{4},{ }^{4}$ Amir Lerman, ${ }^{1}$ and Francisco Lopez-Jimenez $\mathbb{D}^{1}$
}

${ }^{1}$ Department of Cardiovascular Medicine, Mayo Clinic, Rochester, MN, USA

${ }^{2}$ Department of Cardiology, Baylor College of Medicine, Houston, TX, USA

${ }^{3}$ Division of Biostatistics, Mayo Clinic College of Medicine, Rochester, MN, USA

${ }^{4}$ Division of Nephrology and Hypertension, Department of Internal Medicine, Mayo Clinic, Rochester, MN, USA

Correspondence should be addressed to Francisco Lopez-Jimenez; lopez@mayo.edu

Received 4 June 2019; Revised 2 October 2019; Accepted 5 February 2020; Published 26 March 2020

Academic Editor: Claire Stocker

Copyright ( 12020 Conor Senecal et al. This is an open access article distributed under the Creative Commons Attribution License, which permits unrestricted use, distribution, and reproduction in any medium, provided the original work is properly cited.

\begin{abstract}
Importance. Obesity is a worsening epidemic worldwide. Effective and accessible weight loss programs to combat obesity on a large scale are warranted, but a need for frequent face-to-face care might impose a limitation. Objective. To evaluate whether individuals following a weight loss program based on a mobile application, wireless scale, and nutritional program but no face-toface care can achieve clinically significant weight loss in a large cohort. Design. Retrospective observational analysis. Setting. China from October 2016 to December 2017. Participants. Mobile application users with a minimum of 2 weights (baseline and $\geq 35$ days). Intervention. A commercial (Weijian Technologies) weight loss program consisting of a dietary replacement, selfmonitoring using a wireless home scale, and frequent guidance via mobile application. Main Outcome. Mean weight change around 42, 60, 90, and 120 days after program initiation with subgroup analysis by gender, age, and frequency of use. Results. 251,718 individuals, with a mean age of 37.3 years (SD: 9.86) (79\% female), were included with a mean weight loss of $4.3 \mathrm{~kg}$ (CI: \pm 0.02 ) and a mean follow-up of 120 days (SD: 76.8 days). Mean weight loss at $42,60,90$, and $120 \mathrm{~d}$ was $4.1 \mathrm{~kg}$ (CI: \pm 0.02 ), $4.9 \mathrm{~kg}$ (CI: \pm 0.02$), 5.6 \mathrm{~kg}(\mathrm{CI}: \pm 0.03)$, and $5.4 \mathrm{~kg}$ (CI: \pm 0.04$)$, respectively. At $120 \mathrm{~d}, 62.7 \%$ of participants had lost at least $5 \%$ of their initial weight. Both genders and all usage frequency tertiles showed statistically significant weight loss from baseline at each interval $(P<0.001)$, and this loss was greater in men than in women $(120 \mathrm{~d}: 6.5 \mathrm{vs.} 5.2 \mathrm{~kg} ; P<0.001)$. The frequency of recording (categorized as high-, medium-, or low-frequency users) was associated with greater weight loss when comparing high, medium, and low tertile use groups at all time intervals investigated (e.g., $120 \mathrm{~d}:-8.6,-5.6$, and $-2.2 \mathrm{~kg}$, respectively; $P<0.001$ ). Conclusions. People following a commercially available hybrid weight loss program using a mobile application, wireless scale, and nutritional program without face-to-face interaction on average achieved clinically significant short- and midterm weight loss. These results support the implementation of comparable technologies for weight control in a large population.
\end{abstract}

\section{Introduction}

Obesity is a worldwide epidemic that continues to grow in scale [1]. It is closely related to many disease states, including cardiovascular disease, diabetes, and several forms of malignancy [2]. Even moderate weight loss (5\% of body weight) can translate to meaningful disease prevention [3]. Effective lifestyle programs have been developed to combat obesity; however, these programs are difficult to scale and are costly and location-specific [4]. Digital health weight loss programs have attractive features of easy dissemination, relatively low cost, and scalability [5]. If these tools are able to effectively facilitate weight loss in individuals, they could be scaled to help curb the obesity epidemic that has coincided with a dramatic rise in smartphone use and Internet connectivity [6].

A number of approaches using mobile and web-based applications alone or in conjunction with food supplements or replacements have been studied with mixed results [7]. While the majority of studies have shown some evidence of weight loss, they were limited by small sample size and heterogeneity of methods [5, 7]. Specific interventions such 
as smartphone-based applications [8,9] and wireless scales [10] seem to be particularly effective. Further research into specific interventions in large and diverse populations and over extended time periods would further clarify the role of these tools in obesity treatment [11].

Adherence to weight loss programs has previously been shown to be an important predictor of success [12]. Small studies have shown increased adherence with the use of mobile application as opposed to paper-based methods for weight monitoring [13]. However, the association between mobile application adherence and weight loss requires further exploration [8].

The aim of this study was to evaluate the use of digital health platform for weight loss utilizing a mobile application and wireless scale in a large retrospective observational study with attention to differences among demographics and frequency of use.

\section{Methods}

2.1. MetaWell. The MetaWell program (Weijian Technologies Inc., Hangzhou, China) is an entirely remote weight loss program, without face-to-face interaction, that consists of a free mobile application combined with a wireless home scale and nutrition program. The MetaWell application is available in the Google Play and Apple App stores. Upon download, users are prompted to register and provide basic demographic information including age and gender. They are also prompted to purchase the associated wireless scale (\$45), a device that captures body weight and bioimpedance measurements, including water content and fat percent. This investigation was focused on weight change only. The program focuses predominantly on weight loss via diet by providing a low-calorie meal plan centered around meal replacement biscuits supplemented with healthy recipes available in the application. While engaged in the program, participants receive individualized meal plans from the application recommending up to 3 Yufit biscuits daily along with a selection of other recommended foods with the aim of negative caloric balance. The program is tailored based on each participant's basal metabolic requirements. Yufit biscuits are low-calorie, noncaffeinated meal replacements with a low glycemic index. They are available for retail purchase as a part of the weight loss program. Their macronutrient profile is 416 calories per $100 \mathrm{~g}$, with $11.2 \mathrm{~g}$ of protein, $17.5 \mathrm{~g}$ of fat, and $44.4 \mathrm{~g}$ of carbohydrates. Complete nutritional information and sample diet plans provided in the app are detailed in Figure 1. The biscuits have not previously been studied. Direct measures of supplement use during the period studied were unavailable. Activity is encouraged during the program, but no specific exercise program is provided. Users are prompted by the application to weigh themselves on a daily basis via the wireless scale. In the application, participants can see a record of their weight loss progress, as well as a "Health Status Overview" that provides a snapshot of their current health data and optimal measures using a goal BMI of $22.0 \mathrm{~kg} / \mathrm{m}^{2}$. Screen captures of the application are presented in Figure 2. If at the initiation of the program or throughout the course of the program a person body weight reaches a BMI of $<22.0 \mathrm{~kg} / \mathrm{m}^{2}$, the program directs them to maintain weight as opposed to continue weight loss.

2.2. Study Protocol. Using a retrospective design, we investigated the data to determine if active MetaWell users on average achieved weight loss and if any recorded factors were associated with more or less weight loss among program's users. A complete, deidentified record of application users collected by Weijian Technologies Inc. was provided for research purposes. Participants provided electronic consent to have their data used for research purposes. All participants in the study downloaded the free application and purchased the associated wireless scale as part of a commercial weight loss program presumably to engage in weight loss; however, motivation was not assessed. This analysis included adult MetaWell users in China that were at least normal weight defined as a BMI of $18.5 \mathrm{~kg} / \mathrm{m}^{2}$ from October 27, 2016, to December 31, 2017. Subjects also needed to have met a minimum of recorded participation including a baseline weight and a weight at 35 days or beyond. The study design, strategy to analyze the data, actual data analysis, and the writing of the manuscript occurred without company input.

The program directs users to start an initial six-week weight loss program; however, it was commonly continued for longer durations. All users who had a baseline weight and weight recorded around specific time points, 14-day intervals centered on 42, 60, 90, and 120 days, were included in the primary analysis, while all users with a baseline weight and a weight at any time point beyond 35 days were included in the secondary analysis. The sets of users in each time window were not identical. A secondary analysis was performed to investigate average weight at last follow-up that included all individuals with a weight recorded at 35 days or longer. Given that the application is designed to facilitate weight loss, subjects with BMI observations categorized as underweight $\left(\mathrm{BMI} \leq 18.5 \mathrm{~kg} / \mathrm{m}^{2}\right)$ were excluded. Exceptionally high BMI $\left(\geq 80 \mathrm{~kg} / \mathrm{m}^{2}\right)$ observations were also excluded presumed to represent erroneous measurements. To improve the reliability of the analysis and remove outliers, the top and bottom $1 \%$ in regard to weight at baseline were also removed from the analysis. Users with a stated age $<18$ or $>100$ years were also excluded. Because this cohort was largely based in China, all analysis performed relied on the BMI classification in adult Asians by the World Health Organization (low: <18.5; normal: 18.5-23.0; preobese: 23.0-27.5; obese: >27.5) [14]. Subgroups were evaluated based on age, gender, baseline weight status, and frequency of use. The frequency of use was defined by the number of weights recorded for each person during each time interval studied divided by the number of days a person was in that time period. Tertiles of these frequencies were then made within the time period to create high (highest $1 / 3$ of users), medium (middle $1 / 3$ of users), and low (lowest $1 / 3$ of users) frequency of use categories for comparison. Among participants who were overweight or obese at baseline, we calculated percent excess body weight and percent excess body weight loss according to published recommendations [15]. 


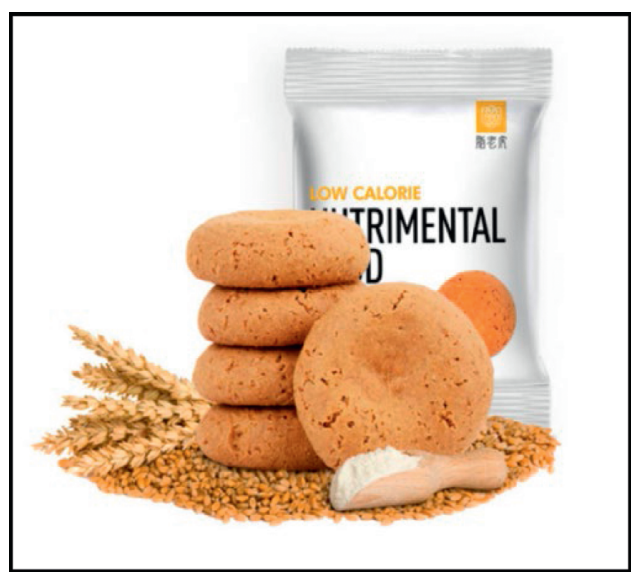

(a)

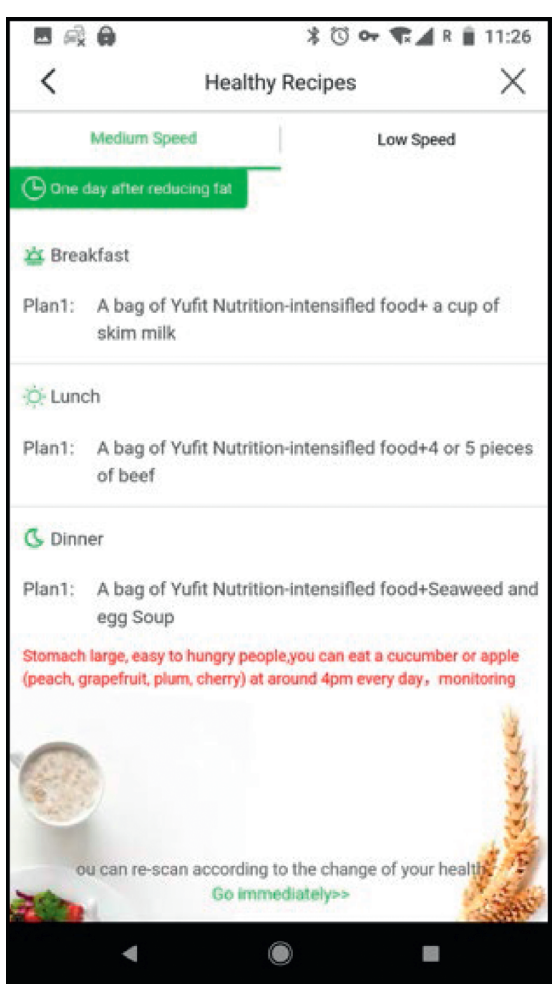

(c)

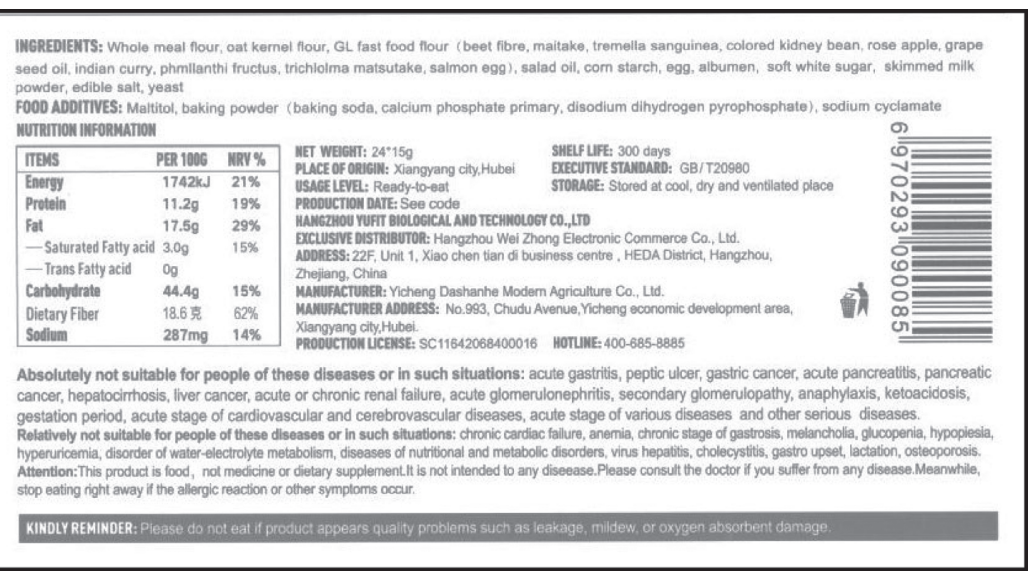

(b)

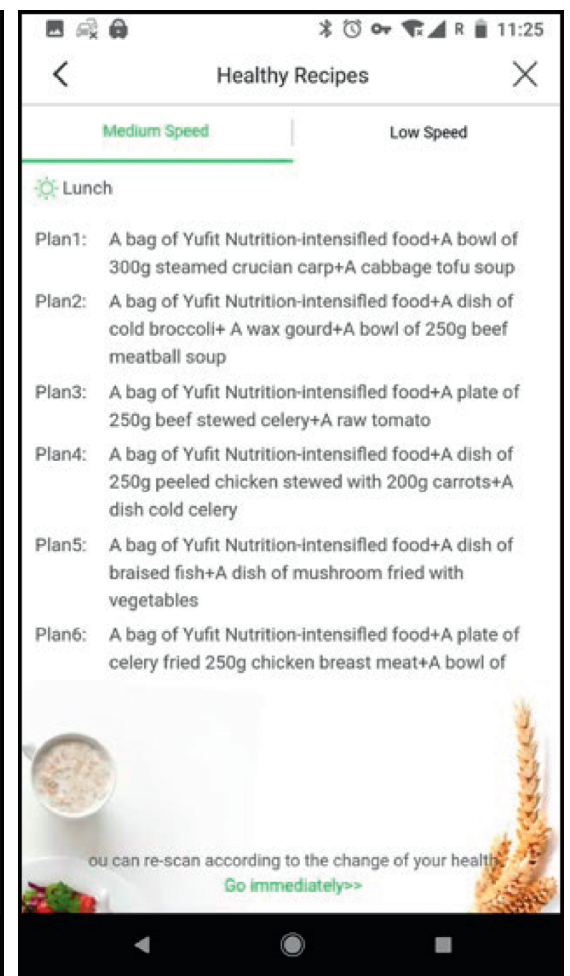

(d)

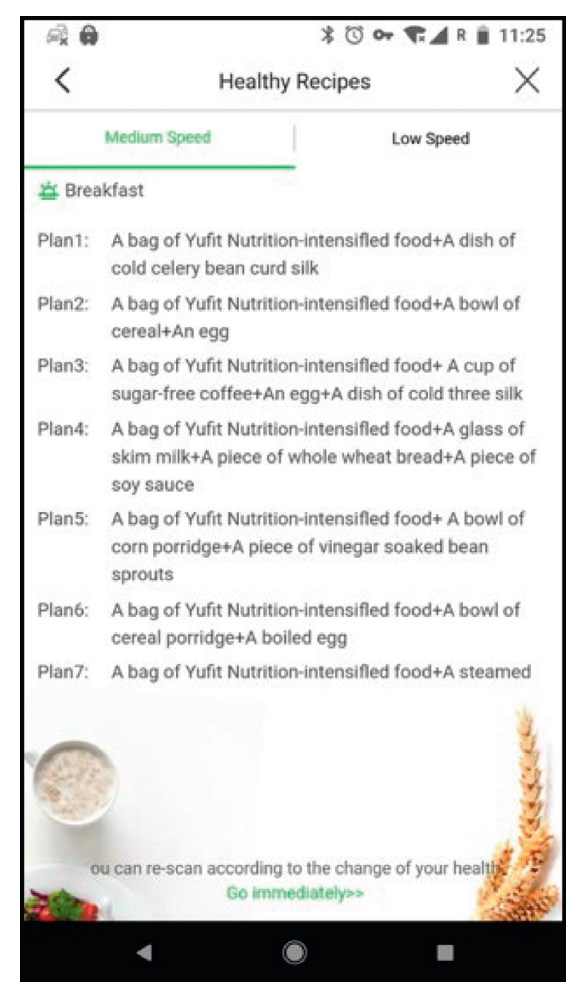

(e)

Figure 1: Nutritional Program. (a) Meal replacement biscuit. (b) Nutritional information provided on meal replacement packaging. (c) Daily summary of nutritional plan. (d) Lunch meal options. (e) Breakfast meal options.

2.3. Statistical Analysis. Means and standard deviations were used to describe continuous variables; counts and percentages were used to summarize categorical variables, both across strata and within stratum. Weight at baseline was determined by taking the median weight within a 3-day period of first user observation. Weights at the end of time intervals were constructed in a similar fashion, but extending the end window to 7 (or 14 in the case of the 120 strata) days before or after the end-date mark. Excess weight was defined as any weight that exceeded that maximum healthy weight as determined by a BMI of 23. Chi-square tests were utilized to investigate if there was an average shift in weights from time 1 to time 2 within stratum, and then further to probe for differences between men and women, age categories, overweight categories, and low-, medium-, or high-frequency weight recorders.

\section{Results}

Overall 251,718 unique individuals, $45 \%$ of the original sample were included (measurement at baseline and a minimum of 35 days), of whom $78.6 \%$ were female. The mean age was $37.3 \pm(9.9)$, and $73.4 \%$ of the population studied was overweight or obese, based on BMI at baseline. 232,759 unique individuals were included in the interval analysis (42-, 60-, 90-, or 120-day interval groups), $78.8 \%$ 


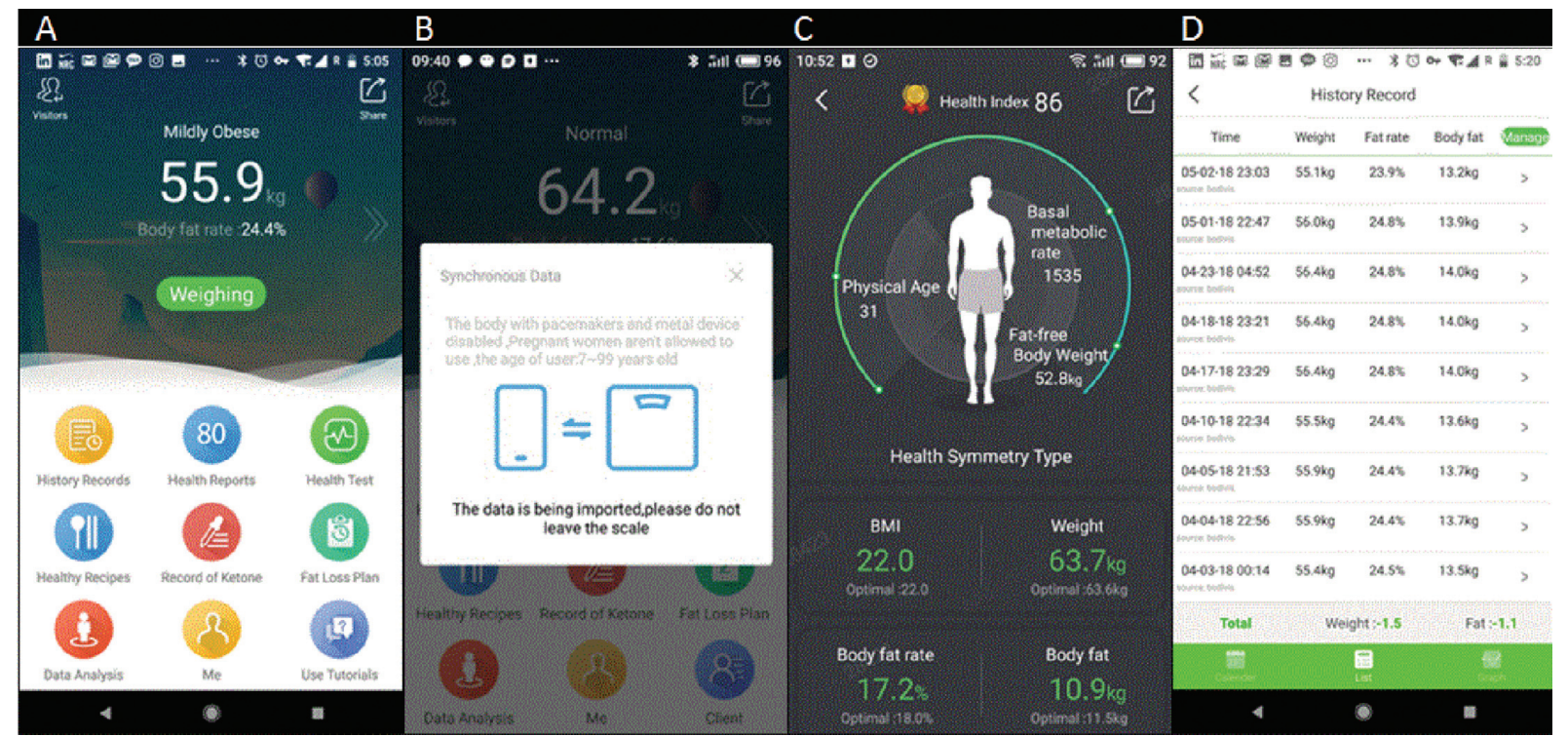

Figure 2: Screenshots of the MetaWell mobile application. (a) Home screen of the application. (b) Weighing screen, shown as users are on scale. (c) Health summary screen. (d) Record of weight change.

female with a mean age of $37.4 \pm(9.9)$. Baseline demographics are shown in Table 1 for individuals included who had met inclusion criteria and who had recorded participation that spanned at least 35 days.

Overall, users had a mean weight loss of $4.3 \mathrm{~kg}$ (CI: \pm 0.02 ) and mean follow-up of 120 days (SD: 76.8 days). In the 42-day period, 192,405 individuals were included (Table 2). Mean weight loss for the population was $4.1 \mathrm{~kg}$ (95\% CI: 0.02 ) with $58.2 \%$ losing $>5 \%$ of their baseline weight. Men on average lost a mean of $5.2 \mathrm{~kg}$ (CI: \pm 0.05 ), which was significantly more than in women, who lost a mean of $3.8 \mathrm{~kg}(\mathrm{CI}: \pm 0.02, P<0.001)$. All age groups lost weight at 42 days, with those $<30$ years of age losing a clinically small, but statistically significant, greater amount than the other age groups studied $(P<0.0001)$. At every interval studied, men lost significantly more weight than women (Table 2, $P<0.001$ for all comparisons). Although absolute weight loss was greater in men, the weight loss relative to their baseline weight or excess weight was greater in women than in men. However, in all tested intervals of 60 days or more, a greater proportion of women than men achieved over $5 \%$ weight loss $(P<0.0001$ for all comparisons). All age groups lost weight at 42 days, but those $<30$ years of age had a slightly higher but statistically significant weight loss, when compared to the other age groups $(P<0.0001)$.

High-frequency users recorded an average of 1.2 (SD: 0.41 ) weight measurements per person day, significantly more than medium ( 0.76 weights/person day, SD: 0.12 ) and low (0.33 weights/person day, SD: 0.16$)$ at the 42 -day interval $(P<0.001$ for all comparisons). Weight loss by the frequency of weight measurement at each time interval is shown in Figure 3. High-frequency users had greater weight loss than medium-frequency users, who had greater weight loss than low-frequency users at each time interval studied $(P<0.001$ for all comparisons).
TABLE 1: Baseline characteristics of the application users.

\begin{tabular}{lc}
\hline$N$ & 251,718 \\
Age (years) (SD) & $37.3(9.86)$ \\
Female (\% of total) & $197,854(78.6 \%)$ \\
Baseline weight $(\mathrm{kg})(\mathrm{SD})$ & $69.2(14.5)$ \\
Baseline BMI $\left(\mathrm{kg} / \mathrm{m}^{2}\right)(\mathrm{SD})$ & $25.9(4.19)$ \\
Classification $\left(\mathrm{BMI}, \mathrm{kg} / \mathrm{m}^{2}\right)$ & $N(\%$ of total) \\
Normal [18.5 23) & $67,047(26.6 \%)$ \\
Overweight [23 25) & $53,205(21.1 \%)$ \\
Preobese [25 30) & $93,441(37.1 \%)$ \\
Obesity class I [30 35) & $29,750(12 \%)$ \\
Obesity class II [35 40) & $6,575(2.61 \%)$ \\
Obesity class III [>40) & $1,700(0.675 \%)$ \\
\hline
\end{tabular}

Among users who recorded at least one weight measurement at 35 days or beyond, the mean duration of app use was 120 days (CI: \pm 0.3 ), median 97 days. Women tended to participate longer (mean: 121 days; CI: \pm 0.34 ) than men (mean: 115 days; CI: $\pm 0.64 ; P<0.001$ ). Increased frequency was also associated with longer duration of use. At 120 days, $62.7 \%$ of participants had lost at least $5 \%$ of their initial weight.

Table 3 displays the weight loss and BMI changes among the 172,464 individuals classified as being either overweight or obese at baseline based on a BMI of $\geq 23.0 \mathrm{~kg} / \mathrm{m}^{2}$ (Table 3). Among these, all BMI groups lost significant weight at each interval studied. In general, the groups with the highest excess weight at baseline lost more weight through the intervals studied and similarly had a greater excess weight loss described as a percentage. Of the participants classified as obese at baseline $(38,025), 53 \%$ moved into the overweight or preobese category by the last observed weight, with $2 \%$ moving into a normal BMI category. Of participants categorized as overweight at baseline (146, 646), 30\% achieved a normal BMI categorization by the last observed weight. 
TABLE 2: Weight loss results at specified time intervals by total groups and demographic subgroups.

\begin{tabular}{|c|c|c|c|c|c|c|}
\hline & Total & Men & Women & Age $<30$ & Age $30-50$ & Age $>50$ \\
\hline \multicolumn{7}{|c|}{ Weight loss $(\mathrm{kg})( \pm \mathrm{MOE})$} \\
\hline 42 days & $-4.1(0.02)$ & $-5.2(0.05)$ & $-3.8(0.02)$ & $-4.3(0.04)$ & $-4.1(0.02)$ & $-4.1(0.05)$ \\
\hline 60 days & $-4.9(0.02)$ & $-6.2(0.07)$ & $-4.6(0.02)$ & $-5.1(0.06)$ & $-4.9(0.03)$ & $-4.8(0.06)$ \\
\hline 90 days & $-5.6(0.03)$ & $-6.9(0.10)$ & $-5.3(0.03)$ & $-5.9(0.09)$ & $-5.5(0.04)$ & $-5.4(0.09)$ \\
\hline 120 days & $-5.4(0.04)$ & $-6.5(0.11)$ & $-5.2(0.04)$ & $-5.6(0.10)$ & $-5.4(0.05)$ & $-5.4(0.10)$ \\
\hline \multicolumn{7}{|c|}{$>5 \%$ total body loss, $N$ ( $\%$ of total $)$} \\
\hline 42 days & $112075(58.2 \%)$ & $23432(57.9 \%)$ & $88643(58.3 \%)$ & $24657(58.3 \%)$ & $74128(58.3 \%)$ & $13290(58.1 \%)$ \\
\hline 60 days & $93047(64.1 \%)$ & $18594(62.8 \%)$ & $74453(64.4 \%)$ & $19132(63 \%)$ & $62570(64.4 \%)$ & $11345(64.4 \%)$ \\
\hline 90 days & $61862(65.9 \%)$ & $11604(63.1 \%)$ & $50258(66.5 \%)$ & $11781(63.4 \%)$ & $42320(66.3 \%)$ & $7761(67.4 \%)$ \\
\hline 120 days & $52800(62.7 \%)$ & $9623(59 \%)$ & $43177(63.6 \%)$ & $10092(59.7 \%)$ & $36195(63 \%)$ & $6513(65.7 \%)$ \\
\hline \multicolumn{7}{|c|}{ BMI loss $\left(\mathrm{kg} / \mathrm{m}^{2}\right)( \pm \mathrm{MOE})$} \\
\hline 42 days & $-1.5(0.01)$ & $-1.8(0.02)$ & $-1.5(0.01)$ & $-1.6(0.01)$ & $-1.5(0.01)$ & $-1.5(0.02)$ \\
\hline 60 days & $-1.8(0.01)$ & $-2.1(0.02)$ & $-1.8(0.01)$ & $-1.9(0.02)$ & $-1.8(0.01)$ & $-1.8(0.02)$ \\
\hline 90 days & $-2.1(0.01)$ & $-2.3(0.03)$ & $-2.0(0.01)$ & $-2.2(0.03)$ & $-2.1(0.01)$ & $-2.1(0.03)$ \\
\hline 120 days & $-2.0(0.01)$ & $-2.2(0.04)$ & $-2.0(0.02)$ & $-2.1(0.04)$ & $-2.0(0.02)$ & $-2.1(0.04)$ \\
\hline
\end{tabular}

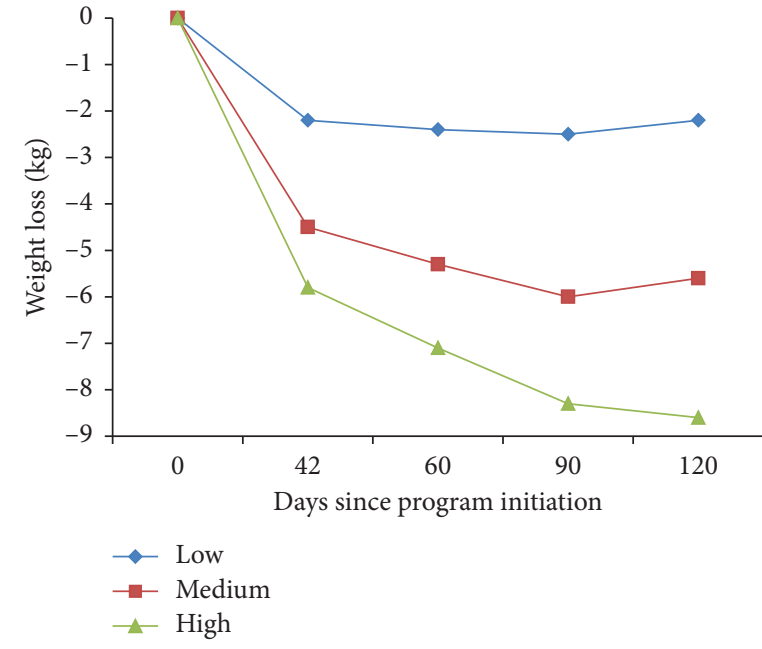

(a)

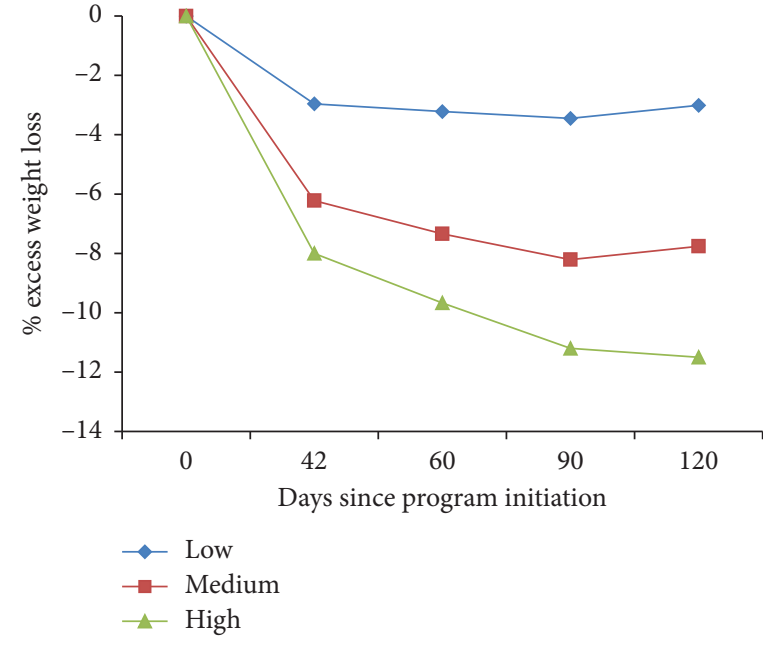

(b)

Figure 3: Weight loss results for application users by the frequency of application; tertiles of these frequencies were then made within the time period to create high (highest $1 / 3$ of users), medium (middle $1 / 3$ of users), and low (lowest $1 / 3$ of users) frequency of use categories for comparison. Significant differences were found among all groups at all intervals shown $(P<0.001)$.

\section{Discussion}

In this large observational study of greater than 250,000 individuals, people following a commercially available weight loss program using a digital health platform without face-to-face interaction and a simple meal supplement achieved clinically significant short- and midterm weight loss. Individuals defined as obese at baseline, thus most likely to benefit from weight loss, [16] experienced greater weight loss. The scale and duration of this study contribute to the growing evidence base that mobile applications may be a useful tool in combating obesity at a population level with the distinct advantage of being widely accessible, relatively low cost, and without the constraint of face-to-face interaction [5].

This is the largest observational study of a mobile application for weight loss with the largest similar study reporting on approximately 36,000 individuals [17]. Our study differs significantly by adding the hybrid approach use of a wireless home scale and specific nutrition plan. Similar to several randomized trials and other observational studies, this study shows an association between weight loss and application use [18]. It is encouraging that significant weight loss was seen in both genders and all age groups studied, suggesting wide applicability. It is also notable that the program did not involve any specific activity recommendations. This highlights the possible potential in groups with limited mobility. It also allows a possible opportunity for continued evolution and improvement in the program.

The magnitude of the weight loss is highly significant, even in the setting of observational research, with $62.7 \%$ of the 120 -day cohort losing $>5 \%$ of their body weight and an average weight loss of $5.4 \mathrm{~kg}$ at 120 days. A systematic review of randomized control trials using in-person interventions 
TABLE 3: Weight loss results for users with $B M I \geq 23.0 \mathrm{~kg} / \mathrm{m}^{2}$.

\begin{tabular}{|c|c|c|c|c|c|}
\hline & $\begin{array}{l}\text { Overweight } \\
(23 \sim 25)\end{array}$ & $\begin{array}{l}\text { Preobese } \\
(25 \sim 30)\end{array}$ & $\begin{array}{l}\text { Obesity class I } \\
\quad(30 \sim 35)\end{array}$ & $\begin{array}{l}\text { Obesity class II } \\
\quad(35 \sim 40)\end{array}$ & $\begin{array}{c}\text { Obesity class III } \\
(>40)\end{array}$ \\
\hline \multicolumn{6}{|l|}{ Baseline } \\
\hline$N$ & 49298 & 87362 & 27993 & 6199 & 1612 \\
\hline BMI (SD) & $24(0.57)$ & $27.1(1.40)$ & $31.9(1.37)$ & $36.9(1.36)$ & $42.7(2.33)$ \\
\hline Weight (kg) (SD) & $62.9(5.24)$ & $72.9(8.16)$ & $88.3(9.88)$ & $104(11.8)$ & $122(14.4)$ \\
\hline$\% \mathrm{EW}(\mathrm{SD})$ & $4.47(2.31)$ & $15.4(4.32)$ & $28.1(3.03)$ & $37.8(2.25)$ & $46.2(2.78)$ \\
\hline \multicolumn{6}{|l|}{42 days } \\
\hline Weight loss $(\mathrm{kg})( \pm \mathrm{CI})$ & $-3.3(0.03)$ & $-4.8(0.02)$ & $-6.6(0.05)$ & $-7.9(0.14)$ & $-8.9(0.30)$ \\
\hline$\%$ EWL $( \pm \mathrm{CI})$ & $5.37(0.05)$ & $6.64(0.05)$ & $7.46(0.09)$ & $7.6(0.20)$ & $7.35(0.38)$ \\
\hline BMI loss $\left(\mathrm{kg} / \mathrm{m}^{2}\right)( \pm \mathrm{CI})$ & $-1.3(0.01)$ & $-1.8(0.01)$ & $-2.4(0.02)$ & $-2.8(0.05)$ & $-3.2(0.12)$ \\
\hline \multicolumn{6}{|l|}{60 days } \\
\hline Weight loss $(\mathrm{kg})( \pm \mathrm{CI})$ & $-3.9(0.04)$ & $-5.8(0.03)$ & $-8.1(0.07)$ & $-9.9(0.18)$ & $-11(0.40)$ \\
\hline$\%$ EWL $( \pm \mathrm{CI})$ & $6.25(0.06)$ & $7.94(0.04)$ & $9.22(0.08)$ & $9.5(0.17)$ & $9.41(0.35)$ \\
\hline BMI loss $\left(\mathrm{kg} / \mathrm{m}^{2}\right)( \pm \mathrm{CI})$ & $-1.5(0.01)$ & $-2.2(0.01)$ & $-3.0(0.02)$ & $-3.5(0.06)$ & $-4.1(0.15)$ \\
\hline \multicolumn{6}{|l|}{90 days } \\
\hline Weight loss $(\mathrm{kg})( \pm \mathrm{CI})$ & $-4.2(0.05)$ & $-6.6(0.05)$ & $-9.8(0.27)$ & $-12(0.27)$ & $-15(0.63)$ \\
\hline$\%$ EWL $( \pm \mathrm{CI})$ & $6.7(0.08)$ & $9.09(0.06)$ & $11.1(0.12)$ & $11.8(0.26)$ & $12.3(0.53)$ \\
\hline BMI loss $\left(\mathrm{kg} / \mathrm{m}^{2}\right)( \pm \mathrm{CI})$ & $-1.6(0.02)$ & $-2.5(0.02)$ & $-3.6(0.04)$ & $-4.4(0.09)$ & $-5.4(0.24)$ \\
\hline \multicolumn{6}{|l|}{120 days } \\
\hline Weight loss $(\mathrm{kg})( \pm \mathrm{CI})$ & $-4(0.06)$ & $-6.6(0.05)$ & $-10(0.13)$ & $-13(0.37)$ & $-16(0.82)$ \\
\hline$\% \mathrm{EWL}( \pm \mathrm{CI})$ & $6.46(0.09)$ & $9.13(0.07)$ & $11.6(0.14)$ & $13(0.35)$ & $13.3(0.68)$ \\
\hline BMI loss $\left(\mathrm{kg} / \mathrm{m}^{2}\right)( \pm \mathrm{CI})$ & $-1.6(0.02)$ & $-2.5(0.02)$ & $-3.7(0.05)$ & $-4.8(0.13)$ & $-5.7(0.30)$ \\
\hline
\end{tabular}

for weight loss including diet, exercise, and meal replacements showed comparable weight loss of 5 to $8.5 \mathrm{~kg}$ at 6 months [19]. Our study shows comparable rates of success but using fewer resources, as our intervention did not require face-to-face interactions or visits to a medical or nutrition facility. Similarly, a recent study showed that an intensive, multifaceted online diabetes prevention program had higher participation but similar weight loss compared to in-person programs [20]. The use of an objective wireless home scale throughout the study, as opposed to selfreporting, adds merit to the weight loss findings [21]. In comparison, the largest available observational mobile app trial noted $31.2 \%$ of users losing $>5 \%$ of baseline weight at 6 months [17], and another large study utilizing a mobile app and scale noted $28.6 \%$ of users with $>5 \%$ at 4 months [22]. The reason for these differences is not clear, but possible factors may include the individualized nutritional plan and nutritional supplement, application design, or increased participant motivation due to financial investment in the program.

Adherence to weight loss interventions has been noted as an important predictor of success [12, 23], a finding which seems to hold true in mobile applications [24]. Our study confirms this finding by showing that users weighing themselves more frequently achieved greater weight loss in a large population, consistent with previous reports $[25,26]$. The duration of this study limits insights into weight change beyond 90 days; however, from the available results, there does appear to be a possible plateau in weight loss during the later stages of the study. Possible mechanisms for weight loss plateau include reduced motivation after initial weight loss, difficulty maintaining the recommended diet for extended periods, or achievement of personal goal weight. Further study evaluating the relationship of weight recording and continued weight loss or weight loss maintenance at extended intervals is warranted.

Obesity is a worldwide epidemic with extensive morbidity and mortality initially centered in developed countries; however, it is accelerating at an alarming rate through the developing world [27]. In addition to the public policy and traditional healthcare mechanisms, new tools will be needed to combat obesity at large scale in relatively lowincome populations. Digital health programs offer a promising addition to this toolset by leveraging the rise in smartphone connectivity to create scalable solutions to help curb the rising obesity epidemic [6].

4.1. Limitations. This study is limited by its observational design which does not allow for a casual association between application use and weight loss. The lack of comparison to a control group is a major limitation because we cannot rule out that motivated people would be able to lose weight on their own while monitoring their body weight regularly. However, epidemiologic and trial data have historically shown that people would rarely lose weight or maintain some weight off without the help of a weight loss program. A randomized control trial would add significantly as would complete follow-up data on all participants. Multiple factors contribute to weight loss [28], but during this study only application usage was evaluated. Further investigation into diet, physical activity, and motivation would be useful additions to future studies. Lack of detailed information regarding the use of the meal supplements limits our ability to analyze the relationship between the use of those supplements and successful weight loss. Extended duration of follow-up would allow for further investigation into the possible prolonged benefits of 
application use. Information gathered for the study is inherently user generated via the app and thus is likely prone to some error. Failure to self-report weight information is likely confounded with weight loss and is a potential source of bias. Efforts were made to remove seemingly physiologically implausible information while retaining as many users as was feasible. The association between usage of the app and weight loss could be explained by inherent features of people likely to use the app more often (being more motivated, disciplined, or engaged). The study population is based in China and likely composed largely of those of Asian descent; further investigation into other ethnic populations should be considered in future research.

\section{Conclusion}

This study shows clinically significant weight loss among a large Chinese population using a mobile application and wireless scale as a part of a commercially available weight loss program. Greater weight loss was seen in users who weighed more frequently and had elevated baseline BMI.

\section{Data Availability}

The weight loss data used to support the findings of this study are available from the corresponding author upon request.

\section{Additional Points}

Question. Is a remote weight loss solution combining a mobile application, wireless scale, and nutritional program with meal replacements effective in a large cohort? Findings. Participants remaining in the program lost on average $4.1 \mathrm{~kg}$ of weight at 42 days and $5.4 \mathrm{~kg}$ at 120 days. Meaning. A remote weight loss solution may provide a viable weight loss solution at scale.

\section{Disclosure}

The company had no influence in the study design, analysis, and interpretation of data.

\section{Conflicts of Interest}

Drs. Amir and Lilach Lerman are serving as consultants to Weijian Technologies Inc., Hangzhou, China.

\section{Acknowledgments}

This work was funded by an unrestricted research grant from Weijian Technologies Inc., Hangzhou, China.

\section{References}

[1] L. Abarca-Gómez, Z. A. Abdeen, Z. A. Hamid et al., "Worldwide trends in body-mass index, underweight, overweight, and obesity from 1975 to 2016: a pooled analysis of 2416 population-based measurement studies in 128.9 million children, adolescents, and adults," Lancet (London, England), vol. 390, no. 10113, pp. 2627-2642, 2017.

[2] National Center for Health Statistics, Health, United States, 2015: With Special Feature on Racial and Ethnic Health Disparities, National Center for Health Statistics (US), Hyattsville, MD, USA, 2016.

[3] C. Ma, A. Avenell, M. Bolland et al., "Effects of weight loss interventions for adults who are obese on mortality, cardiovascular disease, and cancer: systematic review and metaanalysis," BMJ, vol. 359, Article ID j4849, , 2017.

[4] S. B. Heymsfield and T. A. Wadden, "Mechanisms, pathophysiology, and management of obesity," New England Journal of Medicine, vol. 376, no. 3, pp. 254-266, 2017.

[5] N. N. Bhardwaj, B. Wodajo, K. Gochipathala, D. P. Paul, and A. Coustasse, "Can mHealth revolutionize the way we manage adult obesity?," Perspectives in Health Information Management, vol. 14, p. 1a, 2017.

[6] J. Pouschter, Smartphone Ownership and Internet Usage Continues to Climb in Emerging Economies, Pew Research, Washington, DC, USA, 2016.

[7] A. Sorgente, G. Pietrabissa, G. M. Manzoni et al., "Web-based interventions for weight loss or weight loss maintenance in overweight and obese people: a systematic review of systematic reviews," Journal of Medical Internet Research, vol. 19, no. 6, Article ID e229, 2017.

[8] M. C. Carter, V. J. Burley, C. Nykjaer, and J. E. Cade, “Adherence to a smartphone application for weight loss compared to website and paper diary: pilot randomized controlled trial," Journal of Medical Internet Research, vol. 15, no. 4, p. e32, 2013.

[9] C. K. Martin, A. C. Miller, D. M. Thomas, C. M. Champagne, H. Han, and T. Church, "Efficacy of SmartLoss ${ }^{\text {SM }}$, a smartphone-based weight loss intervention: results from a randomized controlled trial," Obesity, vol. 23, no. 5, pp. 935-942, 2015.

[10] D. M. Steinberg, D. F. Tate, G. G. Bennett, S. Ennett, C. Samuel-Hodge, and D. S. Ward, "The efficacy of a daily selfweighing weight loss intervention using smart scales and e-mail," Obesity, vol. 21, no. 21, pp. 1789-1797, 2013.

[11] A. Afshin, D. Babalola, M. McLean et al., "Information technology and lifestyle: a systematic evaluation of internet and mobile interventions for improving diet, physical activity, obesity, tobacco, and alcohol use," Journal of the American Heart Association: Cardiovascular and Cerebrovascular Disease, vol. 5, no. 9, Article ID e003058, 2016.

[12] L. E. Burke, J. Wang, and M. A. Sevick, "Self-monitoring in weight loss: a systematic review of the literature," Journal of the American Dietetic Association, vol. 111, no. 1, pp. 92-102, 2011.

[13] J. Wang, Y. J. Liu, D. P. Lu, and Y. P. Deng, "Research on green degree evaluation method in shipbuilding process," Dalian Ligong Daxue Xuebao/Journal of Dalian University of Technology, vol. 52, no. 2, pp. 221-226, 2012.

[14] W. H. Organization, The Asia-Pacific perspective: redefining obesity and its treatment, Health Communications Australia, Sydney, Australia, 2000.

[15] S. A. Brethauer, J. Kim, M. Chaar et al., "Standardized outcomes reporting in metabolic and bariatric surgery," Obesity Surgery, vol. 25, no. 4, pp. 587-606, 2015.

[16] F. Magkos, G. Fraterrigo, J. Yoshino et al., "Effects of moderate and subsequent progressive weight loss on metabolic function and adipose tissue biology in humans with obesity," Cell Metabolism, vol. 23, no. 4, pp. 591-601, 2016.

[17] S. O. Chin, C. Keum, J. Woo et al., "Successful weight reduction and maintenance by using a smartphone application 
in those with overweight and obesity," Scientific Reports, vol. 6, Article ID 34563, 2016.

[18] G. Flores Mateo, E. Granado-Font, C. Ferré-Grau, and X. Montaña-Carreras, "Mobile phone apps to promote weight loss and increase physical activity: a systematic review and meta-analysis," Journal of Medical Internet Research, vol. 17, no. 11, p. e253, 2015.

[19] M. J. Franz, J. J. VanWormer, A. L. Crain et al., "Weight-loss outcomes: a systematic review and meta-analysis of weightloss clinical trials with a minimum 1-year follow-up," Journal of the American Dietetic Association, vol. 107, no. 10, pp. 1755-1767, 2007.

[20] T. Moin, L. J. Damschroder, M. AuYoung et al., "Results from a trial of an online diabetes prevention program intervention," American Journal of Preventive Medicine, vol. 55, no. 5, pp. 583-591, 2018.

[21] A. L. Bowring, A. Peeters, R. Freak-Poli, M. S. Lim, M. Gouillou, and M. Hellard, "Measuring the accuracy of selfreported height and weight in a community-based sample of young people," BMC Medical Research Methodology, vol. 12, p. 175, 2012.

[22] A. Y. Mao, C. Chen, C. Magana, K. Caballero Barajas, and J. N. Olayiwola, "A mobile phone-based health coaching intervention for weight loss and blood pressure reduction in a national payer population: a retrospective study," JMIR mHealth and uHealth, vol. 5, no. 6, p. e80, 2017.

[23] M. R. Cobain and J. P. Foreyt, "Designing "lifestyle interventions" with the brain in mind," Neurobiology of Aging, vol. 26, no. 1, pp. 85-87, 2005.

[24] S. Jacobs, C. Radnitz, and T. Hildebrandt, "Adherence as a predictor of weight loss in a commonly used smartphone application," Obesity Research \& Clinical Practice, vol. 11, no. 2, pp. 206-214, 2017.

[25] Y. Zheng, L. E. Burke, C. A. Danford, L. J. Ewing, M. A. Terry, and S. M. Sereika, "Patterns of self-weighing behavior and weight change in a weight loss trial," International Journal of Obesity, vol. 40, no. 9, pp. 1392-1396, 2016.

[26] J. J. VanWormer, A. M. Martinez, B. C. Martinson et al., "Selfweighing promotes weight loss for obese adults," American Journal of Preventive Medicine, vol. 36, no. 1, pp. 70-73, 2009.

[27] A. Afshin, M. H. Forouzanfar, M. B. Reitsma et al., "Health effects of overweight and obesity in 195 countries over 25 years," New England Journal of Medicine, vol. 377, no. 1, pp. 13-27, 2017.

[28] K. Elfhag and S. Rossner, "Who succeeds in maintaining weight loss? A conceptual review of factors associated with weight loss maintenance and weight regain," Obesity Reviews, vol. 6, no. 1, pp. 67-85, 2005. 